\title{
Inelastic neutron scattering
}

\author{
Sylvain Petit ${ }^{\mathrm{a}}$ \\ Laboratoire Léon Brillouin, CEA-CNRS, Université Paris-Saclay, CE-Saclay, 91191 \\ Gif-sur-Yvette, France
}

\begin{abstract}
The goal of the JDN22 school was to propose a progressive teaching eager to improve the expertise of students in neutron diffraction. Neutron-based techniques have indeed proved for decades to be essential tools in the investigation of condensed matter. This lecture is however concerned with inelastic neutron scattering and is thus somehow apart. In the context of this school, it should then only be considered as a brief introduction. We give simple examples along with the basics of the spectrometers, and finally useful formula for the inelastic cross sections in different situations. We strongly encourage interested readers to refer to the bibliography for more detailed information.
\end{abstract}

\section{Introduction}

Condensed matter physics has benefited tremendously from both elastic and inelastic neutron scattering techniques for more than half a century. Since then, these techniques have been used to study a wide variety of materials. The main reasons arise from the unique physical properties of the neutron itself, zero electric charge, spin half... Also, since the energies of the neutrons with "thermal" wavelengths match the energy scales of many condensed matter systems, it is possible to use them to probe the dynamics. Excitations that can be studied via neutron scattering range in energy from a few $\mathrm{meV}$ to a fraction of an electron volt. As a result, inelastic neutron scattering provides crucial information about the energetic terms that appear in a model Hamiltonian. While interesting from a pure fundamental point of view, it should be emphasized that these terms also determine the response of these systems to an external perturbation, hence allowing one to predict the functional capabilities of materials.

To give the flavor and introduce the basics of inelastic neutron scattering, it is useful to describe first simple examples, either in the context of lattice dynamics or of spin dynamics.

\subsection{Lattice dynamics}

Let's consider a single mono-atomic chain sketched in Fig. 1(a). Here, the atoms with mass $M$ are at distances $a$, and bound together by springs (constant $\Gamma$ ). In other words, the Hamiltonian describing this system is of the form:

$$
\mathcal{H}=\sum_{n} \frac{1}{2} M \mathbf{v}_{n}^{2}+\frac{1}{2} \Gamma\left(\mathbf{x}_{n+1}-\mathbf{x}_{n}-a\right)^{2}
$$

$\mathbf{v}_{n}$ and $\mathbf{x}_{n}$ are the velocity and position of the individual mass at site labeled $n$. A diffraction experiment on such a sample (if it would exist!) would give Bragg spots at positions such that

\footnotetext{
a e-mail: sylvain.petit@cea.fr
}

(C) The Authors, published by EDP Sciences. This is an Open Access article distributed under the terms of the Creative Commons Attribution License 4.0 (http://creativecommons.org/licenses/by/4.0/). 
$Q_{n}=\frac{2 \pi}{a} n$, characterizing the spatial periodic arrangement of the atoms. Information about the energy scales of the problem, such as inertia $M$ and springs $\Gamma$ is clearly absent.

To get more insight into this question, it is useful to examine the excited states of the chain. Writing the atomic position as $\mathbf{x}_{n}=\mathbf{u}_{n}+n \mathbf{a}$, where the $n a$ are the equilibrium positions, classical mechanics shows that the $u_{n}$ displacements of the atoms are governed by the equations of motion:

$$
M \frac{d^{2} \mathbf{u}_{n}}{d t^{2}}=\Gamma\left(\mathbf{u}_{n+1}-\mathbf{u}_{n}-\mathbf{u}_{n}+\mathbf{u}_{n-1}\right)
$$

whose solutions are plane waves with energy $\omega_{\mathbf{k}}$ and wave-vector $\mathbf{k}$. Introducing these solutions into the above equation, we get the dispersion relation:

$$
-M \omega_{\mathbf{u}}^{2}=\Gamma\left(e^{i \mathbf{k a}}+e^{-i \mathbf{k a}}-2\right)
$$

hence

$$
\omega_{\mathbf{k}}=2 \sqrt{\frac{\Gamma}{M}}\left|\sin \frac{\mathbf{k a}}{2}\right|
$$

This dispersion is a periodic function of $\mathbf{k}$, goes to zero at the Brillouin zone centers and reaches extrema at the zone boundaries. From this simple calculation, we conclude that the excited states of the chain consist in harmonic displacements of the atoms. Energies (frequencies) and wavevectors (wavelength) are related by $\omega_{\mathbf{k}}=2 \sqrt{\frac{\Gamma}{M}}\left|\sin \frac{\mathbf{k a}}{2}\right|$. Quantification of these modes leads to the concept of "phonons", a set of harmonic oscillators, characterized by the energies $\omega_{\mathbf{k}}$ and polarization $\mathbf{e}_{\mathbf{k}}$ (that correspond to the direction of vibrations of the atoms). The displacements $\mathbf{u}_{n}$ are linear combinations of the different phonon modes:

$$
\mathbf{u}_{n}(t)=\sum_{k} e^{i\left(n \mathbf{k} \mathbf{a}-\omega_{\mathbf{k}} k t\right)} \frac{\hbar}{\sqrt{2 M \omega_{\mathbf{k}}}}\left(a_{\mathbf{k}}+a_{-\mathbf{k}}^{+}\right) \mathbf{e}_{\mathbf{k}}
$$

where the $\left\{a_{\mathbf{k}}, a_{\mathbf{k}}^{+}\right\}$are the annihilation and creation phonon operators.

At this point, the question that naturally arises is what would be the outcome of an inelastic neutron scattering experiment on that chain? The response is simple: it would allow one to observe directly the dispersion relation $\omega_{\mathbf{k}}$. Practically, as we will see later, inelastic neutron scattering instruments allow one to define trajectories in the $(\mathbf{k}, \omega)$ space; the number of neutrons recorded in the detector is essentially zero along such trajectories except when they cross the $\omega_{\mathbf{k}}$ map. As shown in Fig. 1(a-d), a series of constant energy or constant Q cuts will, step by step, reconstruct the dispersion. Figure 1f shows an example of such a mapping obtained in the case of PbTe [1] by gathering cuts together: the dispersion becomes visible, above some background. Note that the intensity along the dispersion is not constant and depends on $\mathbf{k}$.

Back to the example of the chain, fitting Eq. (1) to the measured $\omega_{\mathbf{k}}$ would allow to measure $\Gamma$. In some sense, this reasoning is very similar to what we do when we want to analyze diffraction data. Here also the positions or relative orientations of the spins are "measured" based on a model that best fits the diffraction pattern.

\subsection{Spin dynamics}

Let's now consider the example of the two sublattices antiferromagnet depicted in Fig. 2(a). From diffraction alone, the relative orientation of spins is easily determined, but the exchange that couples two neighboring spins, along with the amplitude of a single ion anisotropy term, would be impossible to guess. From inelastic neutron scattering, however, taking advantage of 
(a)

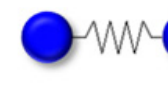

$M$
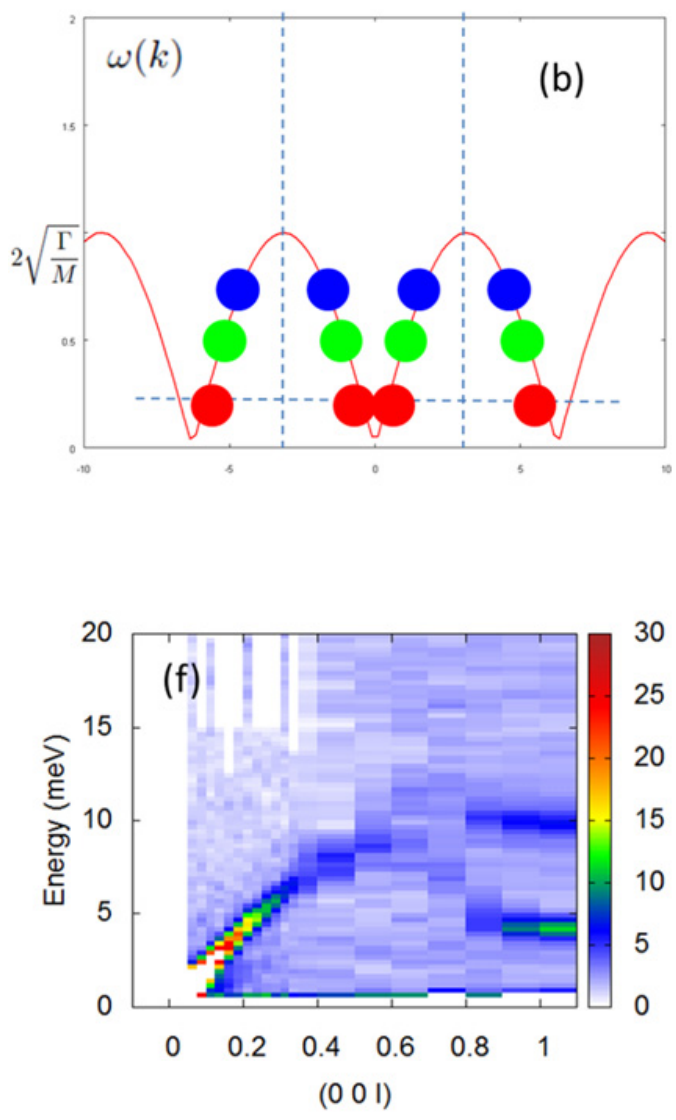
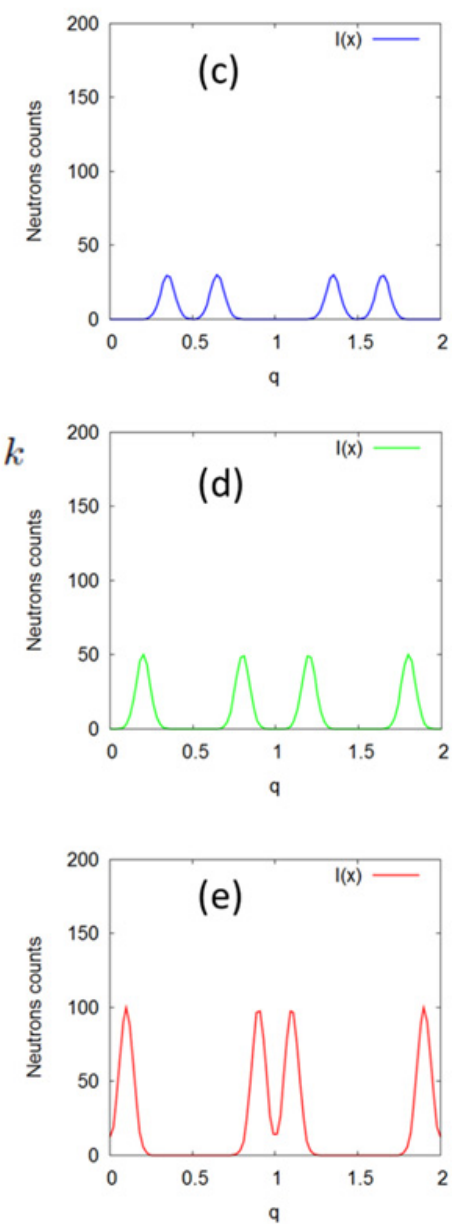

Figure 1. Lattice dynamics of a one 1D chain. (a) Chain model. (b) Shows the dispersion relation, while (c-e) show constant-energy scans across this dispersion. Each peak correspond to a different crossing point in (b). (f) Presents a map of longitudinal phonon propagating along $\mathbf{c}^{*}$ in $\mathrm{PbTe}$, obtained by gathering constant-Q scans together.

the magnetic interaction, it is possible to determine the dispersion of elementary excitations of such a magnet. The latter, called spin waves, are collective excitations that physically correspond to precession motions of the spins around the average direction. From theory, based on the Heisenberg model:

$$
\mathcal{H}=\sum_{i, j} J \mathbf{S}_{i} \cdot \mathbf{S}_{j}+D\left(S_{i}^{z}\right)^{2}
$$

we learn that this dispersion is given by:

$$
\omega_{\mathbf{k}}=S \sqrt{(6 J+D)^{2}-J^{2}\left(2 \cos k_{x} a+2 \cos k_{y} a+2 \cos k_{z} a\right)^{2}}
$$


Figure 2(b) and (c) illustrate these dispersions respectively without and with a single ion anisotropy $D$ that forces the spins to lie down along. In close analogy with the phonon case, a series of constant energy or constant Q cuts will, step by step, reconstruct this dispersion. Fitting it to the above equation will provide numerical values of the exchange $J$ and of the anisotropy $D$. Note especially the presence of a spin gap for $D \neq 0$. Figure 2(d) shows an example of such a mapping obtained in the case of the multiferroic $\mathrm{YMnO}_{3}$ by gathering such cuts together: as for $\mathrm{PbTe}$, the dispersion becomes visible, above some background. Note also that the intensity along the dispersion is not constant and depends on $\mathbf{k}$.

This theory assumes that there is a long range ordering (a phase transition towards a Néel state). In analogy with field theory, the "spin waves" are (bosonic) $\Delta S=1$ particles and the Néel state is considered as the "vacuum" from which these particles emerge. It should be stressed, however, that a number of magnets never order, owing to strong quantum fluctuations, low dimensionality and frustration effects. For instance, recent progress in the physics of low dimensional systems have shown that spin waves are actually made of two spin-half quantum entities called spinons, which under usual circumstances remain confined and thus not visible. Actually, this concept of confinement, when particles are bound together by an interaction whose strength increases with increasing particle separation, is a fundamental idea of modern physics. The most widely studied model in which this phenomenon takes place is the spin-half Heisenberg antiferromagnetic chain with first-neighbor interactions. In agreement with the Wagner and Mermin theorem, quantum fluctuations prevent static long-range order and the ground state remains a spin liquid, with power-law decaying antiferromagnetic correlations. In such a chain, spinons can be seen as domain walls between reversed antiferromagnetic domains; they can move freely and independently along the chain, as no energy cost is involved: in other words, they do not confine. In this case, the magnetic response shows no well-defined spin wave excitations but an incoherent spectrum called two-spinons continuum, which reflects the range of energies corresponding to the different spinon pairs (see Fig. 2(e)).

\section{Principles}

We now turn to the description of spectrometers dedicated to inelastic neutron scattering experiments [2-10]. As for diffraction, most of those experiments demand a monochromatic beam, to be prepared from the white beam produced by the neutron source (reactor or pulsed source). The main idea is then to determine the final energy of the neutrons by analyzing the scattered beam. Taking the energy difference between incident and outgoing neutrons gives the energy $E_{i}-E_{f}=\omega$ given to the sample (note that this quantity can be negative, which correspond to a situation where the neutrons receive energy from the sample and are thus accelerated).

There are essentially two methods for discerning the energy of the neutrons (see Figs. 3 and 4): the first one is by measuring the time it takes for neutrons to travel the distance between the sample to the detector, known as time-of-flight neutron spectroscopy (TOF) [9]. This method assumes that the incident beam is chopped to define the time reference, hence reducing the effective average flux on the sample.

The second method is by Bragg scattering from a single crystal, known as triple-axis spectroscopy (TAS) $[8,10,11]$ :

$$
\lambda=\frac{2 \pi}{\left|\mathbf{k}_{f}\right|}=2 d \sin \theta
$$

It takes advantage of the continuous flux of the source. It was invented by Brockhouse in the mid 1950s at Chalk River [7]. Although there have been many improvements, the principle behind this technique has not changed from that originally developed. The term triple-axis 
(a)
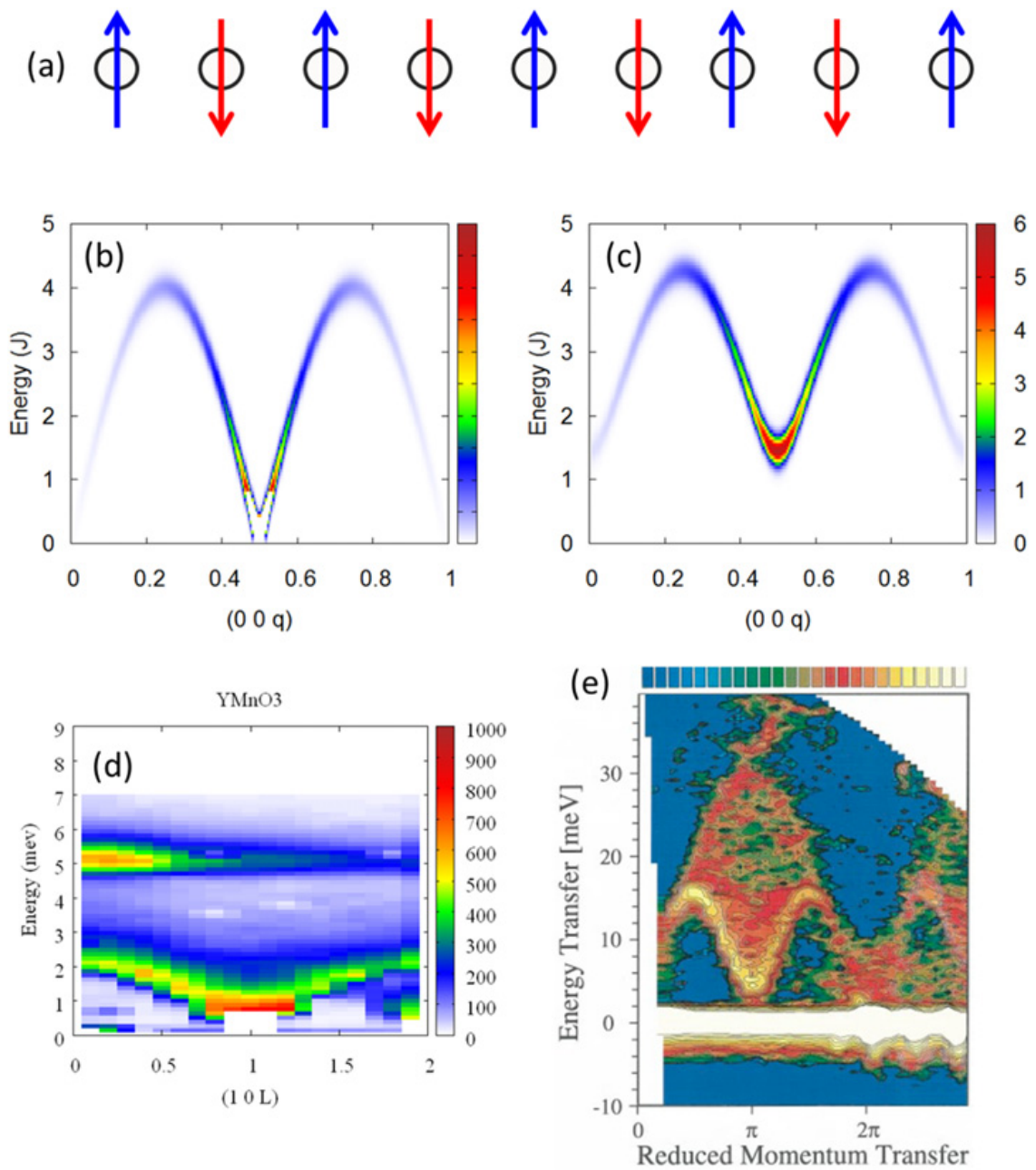

Figure 2. Spin dynamics in a simple two sublattices antiferromagnet. (a) displays the antiferromagnetic chain. (b-c) Show the neutron scattering expected intensity in two different cases, without and with single ion anisotropy respectively. (d) Presents a map of the dispersion of spin waves in the multiferroic material $\mathrm{YMnO}_{3}$ and (e) a map of the two spinons continuum in a 1D chain (M. Arai, M. Fujita, M. Motokawa, J. Akimitsu, and S.M. Bennington, Phys. Rev. Lett. 77, 3649 1996).

derives from the fact that neutrons are scattered from three crystals as they travel from the source to the detector.

Depending on the possible processes that take place in the sample, the scattered neutrons may have a different energy from the incident one. This is sketched in Fig. 3(a) using rays of several colors. At this point, the conservation of energy and momentum writes:

$$
\begin{aligned}
E_{i}-E_{f} & =\omega \\
\mathbf{k}_{i}-\mathbf{k}_{f} & =\mathbf{Q}
\end{aligned}
$$

with neutron incident and final energies and momentum related by:

$$
E_{i, f}=\frac{\hbar^{2} k_{i, f}^{2}}{2 M_{n}}
$$




\section{Source}

(a)

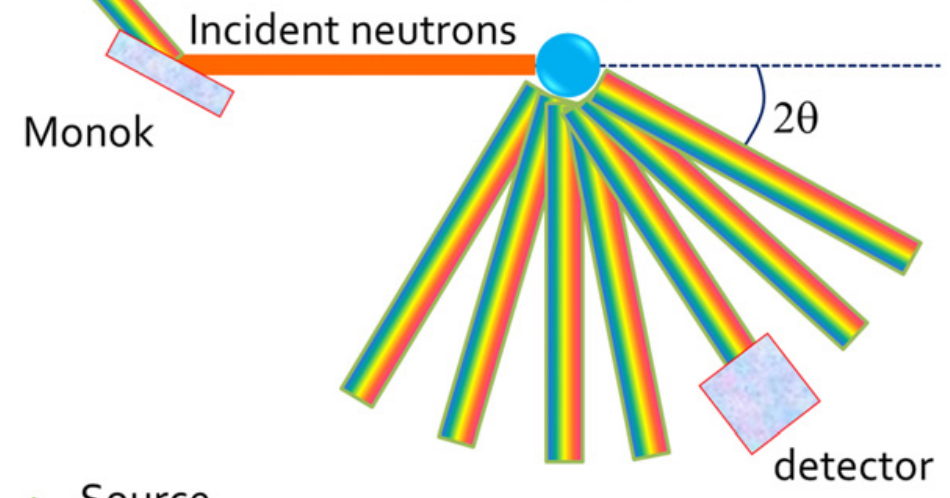

Source

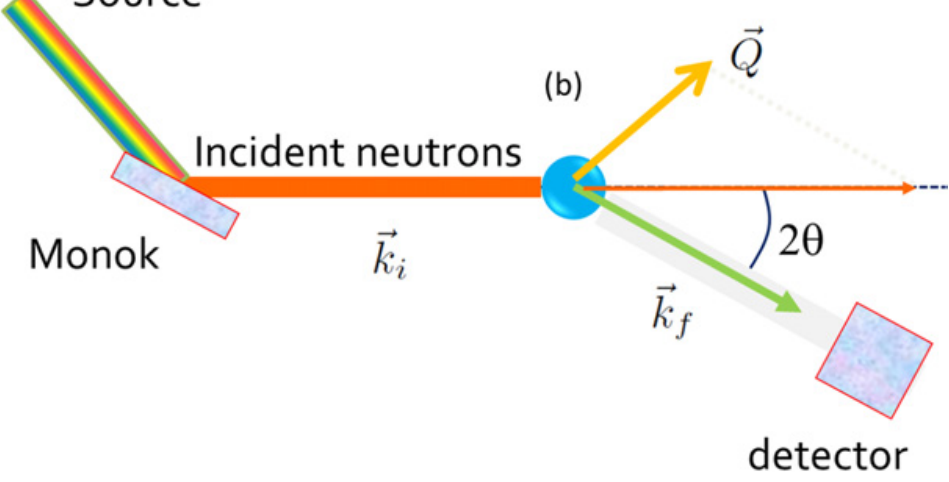

Figure 3. Sketches of a neutron scattering experiment. (a) Pointing out that under a given scattering angle, the beam contains neutrons of different energies; (b) illustrates the conservation of energy and momentum and (c) the rotation necessary to let coincide $\mathbf{Q}$ defined by $\mathbf{k}_{i}-\mathbf{k}_{f}$ and the reciprocal space of the sample.

Importantly, we notice that the scattering angle $\alpha=\left(\mathbf{k}_{i}, \mathbf{k}_{f}\right)$ is determined by the modulus of the wavevector $\mathbf{Q}$ :

$$
\cos \alpha=\frac{k_{i}^{2}+k_{f}^{2}-Q^{2}}{2 k_{i} k_{f}}
$$

The wavevector $\mathbf{Q}$ is determined, for the moment, by $\mathbf{k}_{i}-\mathbf{k}_{f}$, and has no special meaning in terms of reduced reciprocal coordinates $(h, k, \ell)$ of the sample itself. This becomes only true if two particular directions of the sample, within the scattering plane, are known. In that case, $\mathbf{Q}=\mathbf{k}_{i}-\mathbf{k}_{f}$ can be projected onto the sample's reciprocal lattice, so that $(h, k, \ell)$ are uniquely defined. Rotating the sample around the vertical axis allows to address a new set of $\left(h^{\prime}, k^{\prime}, \ell^{\prime}\right)$ coordinates, corresponding to the same modulus.

Each of these methods, TAS or TOF (see Fig. 4(a) and (b) respectively) is most useful in studying a particular type of problem. Traditionally, TAS has been the preferred method for studying single crystals, and TOF for studying powders. Yet, in principle, nothing forbids to measure a single crystal on a TOF instrument. It is just necessary to rotate the sample around the vertical axis, to "reconstruct", for each scattering angle and each time of flight value, the $(h, k, \ell, \omega)$ coordinates. Today, the main advantage of TAS spectrometer consists in the fact 


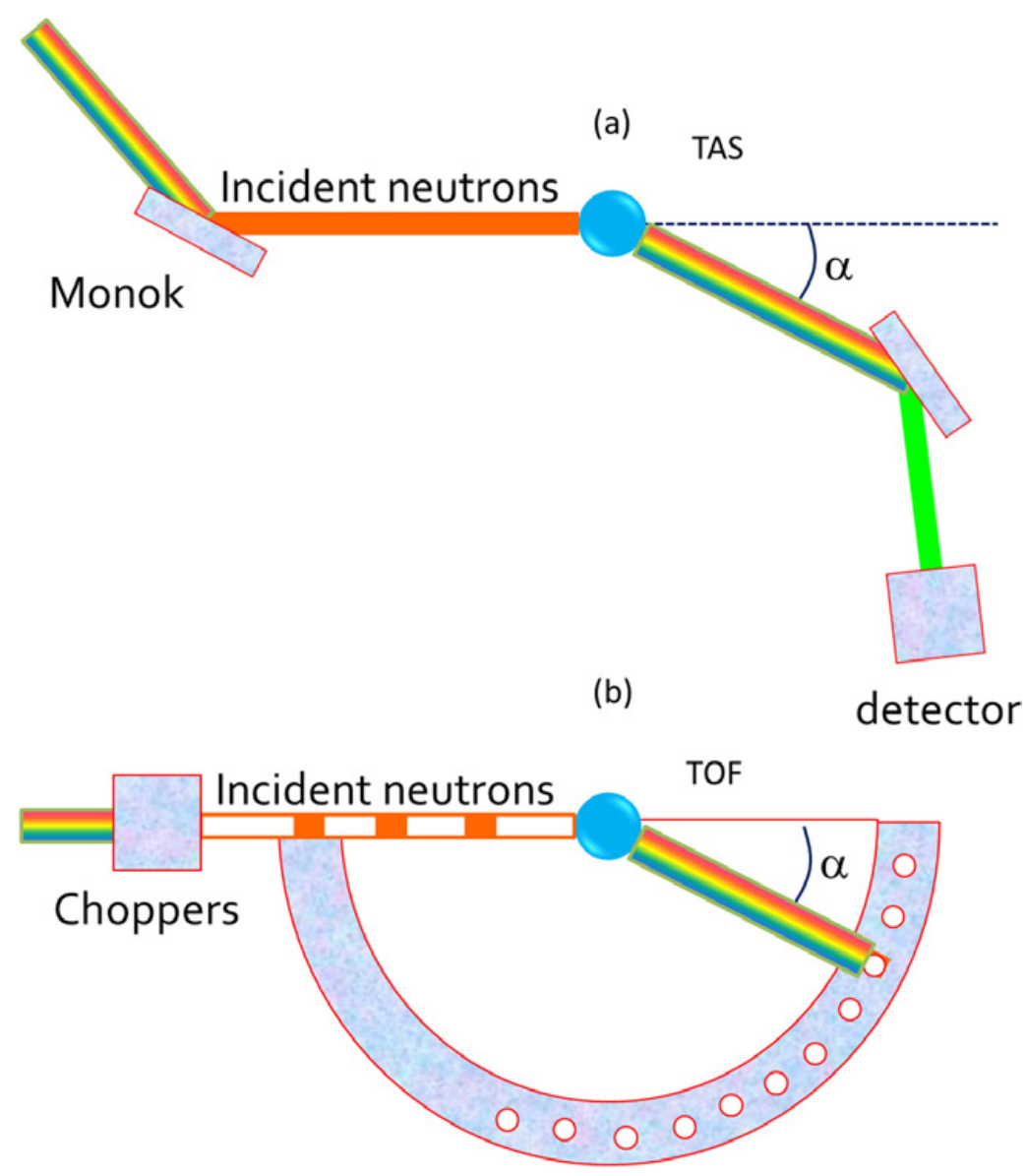

Figure 4. Sketch of TAS and TOF spectrometers.

that it is quite easy to vary external parameters like temperature, magnetic field, pressure or electric field and to measure the response in a small region of $(\mathbf{Q}, \omega)$ space. In contrast, TOF techniques are useful to obtain a survey of excitations in a rather extended $(\mathbf{Q}, \omega)$ region.

\subsection{TAS in practice}

As is clear from the Bragg law, monochromators and analysers select $k_{o}, 2 k_{o}, 3 k_{o} \ldots\left(k_{o}\right.$ standing for $k_{i}$ or a $k_{f}$ ). Even if the measured intensities are normalized to the same number of incident neutrons with the help of a low sensibility detector placed in the incident beam, called "monitor", it remains important to correct these data for those "bad" neutrons with $2 k_{o}, 3 k_{o} \ldots$ This supposes that we know the proportion of harmonics in the incident beam, a quantity that especially depends on the incident energy, the kind of source (thermal, hot, cold, see Fig. 5(a)), and also on the installation of the spectrometer on a neutron guide or not. This correction, called "monitor correction", is shown in Fig. 5(b).

Another issue arising because of harmonics is the possible contamination of the measurement by elastic processes $(\omega=0)$ occurring in the sample provided that:

$$
n k_{o}=m k_{f}(n, m) \text { integers }
$$


(a)

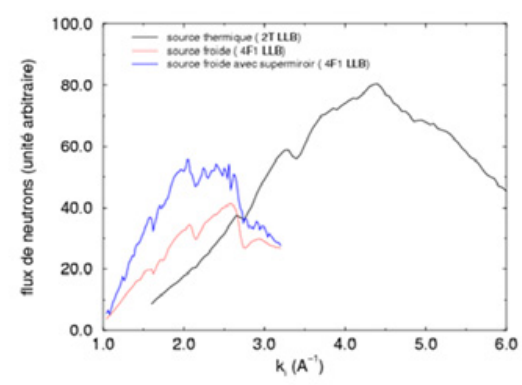

(b)

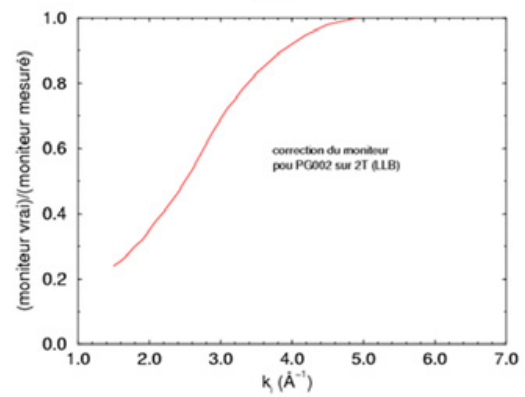

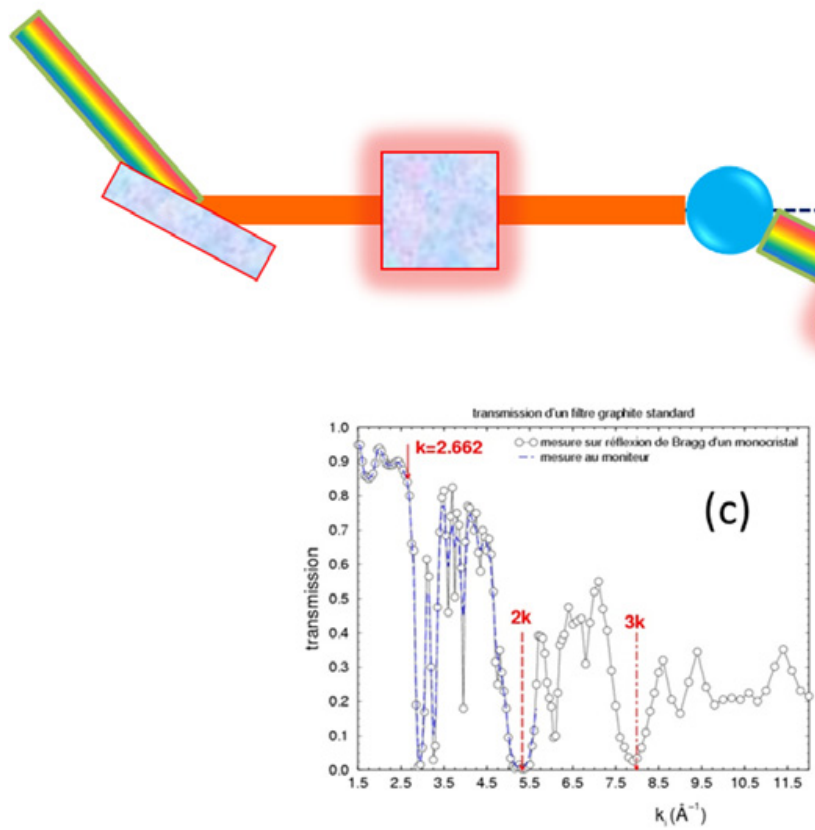

Figure 5. Monitor and filters in a TAS experiment. (a-b) show the incident flux, depending on the kind of source, and the monitor correction obtained for $2 \mathrm{~T}$ [10]. (c) illustrates the filtering with pyrolithic graphite, high transmission for $k_{o}$ and almost zero for the harmonics.

The intensity of such processes can be extremely large compared to the "true" inelastic expected signal. To partly eliminate this problem, filters are used, either placed in the incident or in the scattered beam. The idea is to use a peculiarity of the transmission of pyrolithic Graphite, which is almost 1 for a given $k_{f}$ and 0 for the harmonics, at least (in practice) for $2 k_{f}$. This happens for "magic" values, that are widely used on TAS, 4.1, 3.85, 2.662, 2.57, 1.97, 1.64, and $1.48 \AA^{-1}$ (see Fig. 5(c)). The Beryllium is another material widely used on cold TAS. If cooled at $70 \mathrm{~K}$, its transmission is about 1 for wavevectors lower than $1.55 \AA^{-1}$ and 0 above.

Finally, it is of fundamental importance to introduce the concept of resolution. Actually, a spectrometer cannot isolate a single point in $(Q, \omega)$ space; rather, it integrates over a small volume around that point, an effect which can be modeled by the following convolution:

$$
I(\mathbf{Q}, \omega)=\int d \mathbf{Q}^{\prime} d \omega^{\prime} R\left(\omega-\omega^{\prime}, \mathbf{Q}-\mathbf{Q}^{\prime}\right) S\left(\omega^{\prime}, \mathbf{Q}^{\prime}\right)
$$


(a)

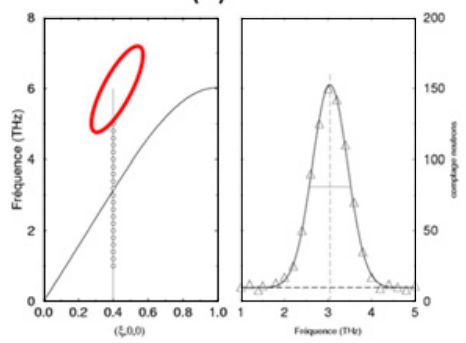

(b)

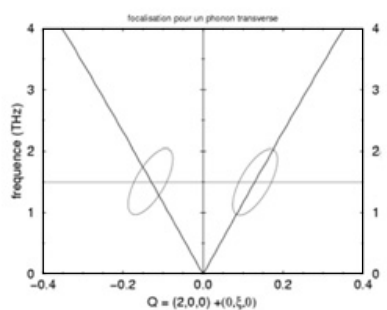

(c)

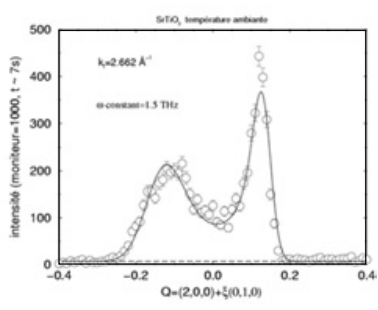

Figure 6. Illustration of the ellipsoid resolution on a TAS [10]. The effect of the inclination of the long axis with respect to the slope of the dispersion is especially illustrated. If these are parallel, then the signal is narrow and intense, while it is broad and weak in the opposite situation. These two possibilities are called focused and unfocused conditions.

with

$$
R(\omega, \mathbf{Q})=V_{i} V_{f} \exp \left[-\{\mathbf{Q}, \omega\}^{T} \bar{A}\{\mathbf{Q}, \omega\}\right]
$$

$\{\mathbf{Q}, \omega\}$ is a 4 dimensional vector with components $\mathbf{Q}$ and $\omega . \bar{A}$ is a $4 \times 4$ matrix. Explicated in its eigen-directions, becomes a nice 4D-ellipsoid with long and short axes, called resolution ellipsoid. A constant energy scans across the dispersion relation gives en enlarged peak as sketched in Fig. 6(a). There exists softwares able to calculate $R$ [10] but we shall give here only guidelines:

- the z-axis can be considered as decoupled from the other quantities. The vertical resolution is generally quite poor.

- because of the inclination of the ellipsoid in $\left(q_{x}, q_{y}, \omega\right)$ space, the analysis of dispersion can become tricky, with especially a focused and an unfocused side: as sketched in Fig. 6(b) and (c), the apparent width of branches can look very different.

- the smaller $k_{f}$ or $k_{i}$, the better the resolution; however, since $V_{i, f} \approx k_{i, f}^{3}$, the less intense is the expected signal.

\section{Cross sections}

The quantity that we ultimately measure is the neutron cross section. It is proportional to the number of neutrons scattered in a solid angle $\Omega$ with a final energy $E_{f}$.

\subsection{Nuclear interaction}

In the case of nuclear interaction, this cross section can be written as follows:

$$
\frac{\partial^{2} \sigma}{\partial \boldsymbol{\Omega} \partial E_{f}}=\frac{k_{f}}{k_{i}} \sum_{i, j} b_{i} b_{j} \int_{-\infty}^{+\infty} d t\left\langle e^{i \mathbf{Q} \mathbf{R}_{i}(t=0)} e^{-i \mathbf{Q} \mathbf{R}_{j}(t)}\right\rangle e^{-i \omega t}
$$

where $b_{i}$ is the scattering length of ion at site $i$ with time dependent position $\mathbf{R}_{i}(t)$.

\subsubsection{The frozen lattice}

Moving in gradual steps, let's first assume that the lattice is frozen, so that these positions do not depend on time. The vector $\mathbf{R}_{i}$ is decomposed into a vector that labels the unit cell $\mathbf{R}_{m}^{o}$ and a second vector within the unit cell $\mathbf{r}_{\ell}$. Concomitantly, the site $i$ is decomposed into two 
new indexes $i=(m, \ell)$.

$$
\mathbf{R}_{i}(t)=\mathbf{R}_{m}^{o}+\mathbf{r}_{\ell}
$$

Note that because of the spatial periodicity, the $b_{i}$ 's only depend on $\ell$. The cross section becomes:

$$
\begin{aligned}
\frac{\partial^{2} \sigma}{\partial \Omega \partial E_{f}} & =\frac{k_{f}}{k_{i}} \delta(\omega) \sum_{m, n, \ell, \ell^{\prime}} b_{\ell} b_{\ell^{\prime}} e^{i \mathbf{Q}\left(\mathbf{R}_{m}^{o}+\mathbf{r}_{\ell}-\mathbf{R}_{n}^{o}-\mathbf{r}_{\ell^{\prime}}\right)} \\
& =\delta(\omega) \sum_{m, n} e^{i \mathbf{Q}\left(\mathbf{R}_{m}^{o}-\mathbf{R}_{n}^{o}\right)} \sum_{\ell, \ell^{\prime}} b_{\ell} b_{\ell^{\prime}} e^{i \mathbf{Q}\left(\mathbf{r}_{\ell}-\mathbf{r}_{\ell^{\prime}}\right)} \\
& =\delta(\omega) \sum_{m, n} e^{i \mathbf{Q}\left(\mathbf{R}_{m}^{o}-\mathbf{R}_{n}^{o}\right)}\left|F_{N}(\mathbf{Q})\right|^{2} \\
& =\delta(\omega) \sum_{\tau} \delta(\mathbf{Q}-\tau)\left|F_{N}(\mathbf{Q})\right|^{2}
\end{aligned}
$$

where $\tau$ is a reciprocal lattice vector and $F_{N}(\mathbf{Q})$ is the familiar nuclear structure factor:

$$
F_{N}(\mathbf{Q})=\sum_{\ell} b_{\ell} e^{i \mathbf{Q} \mathbf{r}_{\ell}}
$$

As a result, in this approximation, the cross section is purely elastic $(\omega=0)$ and consists of Dirac functions at the nodes of the reciprocal lattice. Their intensities are given by the squared modulus of the structure factor.

\subsubsection{The harmonic approximation}

We now turn to the "vibrating" lattice approximation. The $\mathbf{R}_{i}(t)$ become:

$$
\mathbf{R}_{i}(t)=\mathbf{R}_{m}^{o}+\mathbf{r}_{\ell}+\mathbf{u}_{m, \ell}(t)
$$

where $\mathbf{u}_{m, \ell}(t)$ describes the harmonic oscillations, in terms of phonon modes, of the atom labeled $(m, \ell)$. The cross section writes:

$$
\frac{\partial^{2} \sigma}{\partial \Omega \partial E_{f}}=\frac{k_{f}}{k_{i}} \sum_{m, n} e^{j \mathbf{Q}\left(\mathbf{R}_{m}^{o}-\mathbf{R}_{n}^{o}\right)} \sum_{\ell, \ell^{\prime}} b_{\ell} b_{\ell} e^{j \mathbf{Q}\left(\mathbf{r}_{\ell}-\mathbf{r}_{\ell^{\prime}}\right)} \int_{-\infty}^{+\infty} d t\left\langle e^{j \mathbf{Q} \mathbf{u}_{m, \ell}} e^{-i \mathbf{Q} \mathbf{u}_{n, \ell^{\prime}}(t)}\right\rangle e^{i \omega t}
$$

Using quantum properties of the phonon creation and annihilation operators, the expectation value $\langle.$.$\rangle can be rearranged:$

$$
\left\langle e^{i \mathbf{Q} \mathbf{u}_{m, \ell}} e^{-i \mathbf{Q} \mathbf{u}_{n, \ell^{\prime}}(t)}\right\rangle=e^{-W_{\ell}-W_{\ell^{\prime}}} e^{\left\langle\mathbf{Q} \mathbf{u}_{m, \ell} \mathbf{Q} \mathbf{u}_{n, \ell^{\prime}}(t)\right\rangle}
$$

$W_{\ell}$ is the so called Debye-Waller factor, that physically describes the average squared deviations of the ions at temperature $T$ with respect to their equilibrium position:

$$
W_{\ell}=\frac{1}{2}\left\langle\left[\mathbf{Q} \mathbf{u}_{m, \ell}\right]^{2}\right\rangle
$$

The cross section becomes:

$$
\frac{\partial^{2} \sigma}{\partial \Omega \partial E_{f}}=\frac{k_{f}}{k_{i}} \sum_{m, n} e^{i \mathbf{Q}\left(\mathbf{R}_{m}^{o}-\mathbf{R}_{n}^{o}\right)} \sum_{\ell, \ell^{\prime}} b_{\ell} b_{\ell^{\prime}} e^{i \mathbf{Q}\left(\mathbf{r}_{\ell}-\mathbf{r}_{\ell^{\prime}}\right)} e^{-W_{\ell}-W_{\ell^{\prime}}} \int_{-\infty}^{+\infty} d t e^{\left\langle\mathbf{Q} \mathbf{u}_{m, \ell} \mathbf{Q} \mathbf{u}_{n, \ell^{\prime}}(t)\right\rangle} e^{-i \omega t}
$$

Expanding the exponential function to second order, one obtains:

$$
e^{\left\langle\mathbf{Q} \mathbf{u}_{m, \ell} \mathbf{Q} \mathbf{u}_{n, \ell^{\prime}}(t)\right\rangle} \approx 1+\left\langle\mathbf{Q} \mathbf{u}_{m, \ell} \mathbf{Q} \mathbf{u}_{n, \ell^{\prime}}(t)\right\rangle+\ldots
$$


The zeroth order leads to a slight modification of the structure factor which now incorporates the Debye-Waller factor:

$$
\begin{aligned}
\frac{\partial^{2} \sigma}{\partial \Omega \partial E_{f}} & =\delta(\omega) \sum_{\tau} \delta(\mathbf{Q}-\tau)|F(\mathbf{Q})|^{2} \\
F_{N}(\mathbf{Q}) & =\sum_{\ell} b_{\ell} e^{i \mathbf{Q} \mathbf{r}_{\ell}} e^{-W_{\ell}}
\end{aligned}
$$

The second order term is the phonon cross section:

$$
\frac{\partial^{2} \sigma}{\partial \Omega \partial E_{f}}=\sum_{s} A_{s}\left[\left(1+n\left(\omega_{\mathbf{Q}, s}\right)\right) \delta\left(\omega-\omega_{\mathbf{Q}, s}\right)+n\left(\omega_{\mathbf{Q}, s}\right) \delta\left(\omega+\omega_{\mathbf{Q}, s}\right)\right]
$$

and consists in a sum of Dirac functions on the different phonon branches. This finally demonstrates that an inelastic neutron scattering experiment allows a direct measurement of the dispersion relations. The intensity along the dispersion is given by the "dynamical" structure factor:

$$
A_{s}=\left|F_{s}\right|^{2}
$$

with

$$
F_{S}(\mathbf{Q})=\sum_{\ell} b_{\ell} e^{i \mathbf{Q} \mathbf{r}_{\ell}} e^{-W_{\ell}} \sqrt{\frac{\hbar}{M_{\ell} \omega_{q, s}}}\left(\mathbf{Q}_{\mathbf{e}_{q, \ell, s}}\right)
$$

The scalar product between the wavevector $\mathbf{Q}$ and the polarization $\mathbf{e}_{q, \ell, s}$ is especially useful as it allows to distinguish transverse and longitudinal vibrations. Longitudinal phonons correspond to vibrations parallel to the propagation vector $\mathbf{q}$, while transverse phonons correspond to vibrations perpendicular to $\mathbf{q}$. To this end, it suffices to measure in different Brillouin zones. With $\mathbf{Q}=\tau+\mathbf{q}$ ( $\tau$ is the zone center $)$, different configurations can be chosen so that $\mathbf{Q}$ is approximatively parallel or perpendicular to a given polarization. Figure 7(a) shows an example of this trick in the case of $\mathrm{CaF}_{2}$, a sample very often used for practicals at LLB: maps obtained for transverse (Fig. 7(c)) and longitudinal (Fig. 7(d)) phonons propagating along $(h h 0)$ are shown as well as a typical individual Q-scan used to produce the maps.

Note that positive energy processes correspond to the creation of a phonon by a neutron, while negative energy process correspond to the annihilation of a phonon. $n\left(\omega_{\mathbf{Q}, s}\right)$ is the Bose number at temperature $T$ :

$$
n\left(\omega_{\mathbf{Q}, s}\right)=\frac{1}{e^{\frac{\hbar \omega_{Q}, s}{k_{B} T}}-1}
$$

Further, the two processes do not have the same intensity: the creation is always possible, even at low temperature, while the annihilation supposes that the initial quantum state of the sample contains phonons. For instance, the annihilation is forbidden at very low temperature since there are no phonons in the ground state configuration. This is called the "detailed 


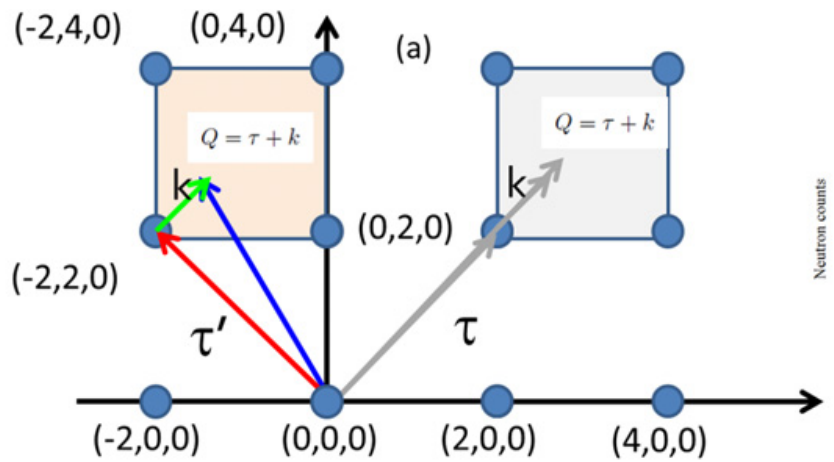

(c) Phonon transwerse

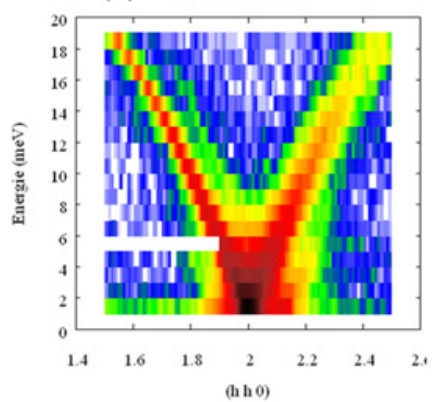

(d)

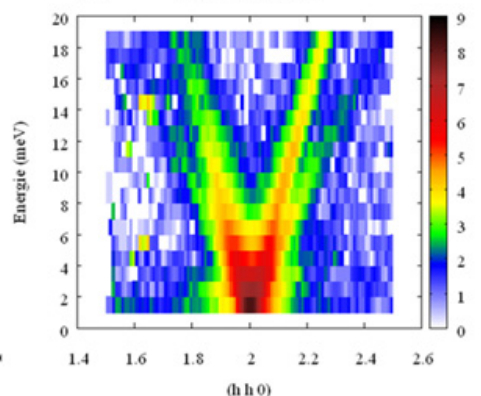

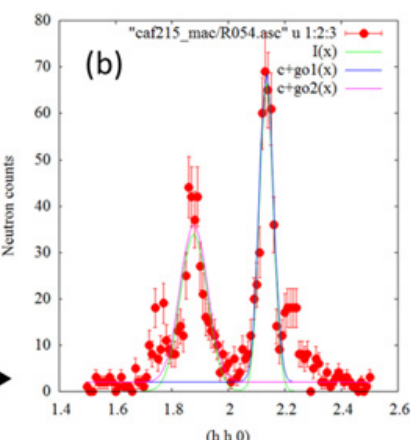

$E=-10 \mathrm{meV}$

Figure 7. Inelastic neutron scattering tutorial in $\mathrm{CaF}_{2}$. (a) Sketch of the reciprocal plane $(h k 0)$, along with two Brillouin zone appropriate fir the study of transverse and longitudinal phonon modes. (b) Shows an individual constant-energy scan while (c) and (d) show the maps obtained by gathering such scans in transverse and longitudinal conditions.

balance"; this is an extremely important concept, also known in other spectroscopy methods like IR or Raman scattering for instance.

\subsubsection{Connection with diffraction}

As already pointed out, a diffraction experiment do not analyze the final energy of the neutrons but rather integrates over these final energies. As a result, this kind of measurement is sensitive to both the pure elastic Bragg scattering, but also to the excitations. As the cross section is very small, it appears as a kind of diffuse scattering, which can be difficult to distinguish from the background. Note that $\omega$ cannot be larger than the incident energy $E_{i}$, so that the corresponding cross section reads:

$$
\frac{\partial \sigma}{\partial \Omega}=\int_{-\infty}^{E_{i}} d \omega \frac{k_{f}}{k_{i}} \sum_{i, j} b_{i} b_{j} \int_{-\infty}^{+\infty} d t\left\langle e^{i \mathbf{Q} \mathbf{R}_{i}} e^{-i \mathbf{Q} \mathbf{R}_{j}(t)}\right\rangle e^{-i \omega t}
$$

If $E_{i}$ is large enough compared to the dynamics, one usually integrates out the $\omega$ dependence to write:

$$
\frac{\partial \sigma}{\partial \Omega}=\sum_{i, j} b_{i} b_{j}\left\langle e^{i \mathbf{Q} \mathbf{R}_{i}(t=0)} e^{-i \mathbf{Q} \mathbf{R}_{j}(t=0)}\right\rangle
$$


In other words, diffraction measures instantaneous correlations. Diffraction at zero energy measures elastic (or infinite time) correlations; inelastic scattering measures the whole (finite time) spectrum of these correlations.

\subsection{Magnetic interaction}

In the case of the magnetic interaction, the cross section is given by:

$$
\frac{\partial^{2} \sigma}{\partial \Omega \partial E_{f}}=\frac{k_{f}}{k_{i}}\left(\gamma_{N} r_{o}\right)^{2} \sum_{i, j} \int_{-\infty}^{+\infty} d t\left\langle\mathbf{S}_{\perp, i} \mathbf{S}_{\perp, j}(t) e^{i \mathbf{Q} \mathbf{R}_{i}} e^{-i \mathbf{Q} \mathbf{R}_{j}(t)}\right\rangle e^{-i \omega t}
$$

$\gamma_{N}=1.913$ is the gyromagnetic ratio of the neutron, $r_{o}$ is the classical electron radius. $\mathbf{S}_{\perp}$ is the component of the spin perpendicular to the scattering vector $\mathbf{Q}$. It is worth noting that:

$$
\mathbf{S}_{\perp, i} \mathbf{S}_{\perp, j}(t)=\sum_{\alpha, \beta} S_{\alpha, i}\left(\delta_{\alpha, \beta}-\frac{Q_{\alpha} Q_{\beta}}{Q^{2}}\right) S_{\beta, j}(t)
$$

The exact derivation of the cross section goes well beyond this lecture; we shall however give the final result:

$$
\begin{aligned}
& \frac{\partial^{2} \sigma}{\partial \boldsymbol{\Omega} \partial E_{f}}=\frac{k_{f}}{k_{i}}\left(\gamma_{N} r_{o}\right)^{2} \sum_{m, n} e^{i \mathbf{Q}\left(\mathbf{R}_{m}^{o}-\mathbf{R}_{n}^{o}\right)} \sum_{\ell, \ell^{\prime}} f_{\ell}(\mathbf{Q}) f_{\ell^{\prime}}^{*}(\mathbf{Q}) e^{i \mathbf{Q}\left(\mathbf{r}_{\ell}-\mathbf{r}_{\ell^{\prime}}\right)} e^{-W_{\ell}-W_{\ell^{\prime}}} \\
& \int_{-\infty}^{+\infty} d t\left\langle\mathbf{S}_{\perp, \ell} \mathbf{S}_{\perp, \ell^{\prime}}(t)\right\rangle e^{-i \omega t}
\end{aligned}
$$

\subsection{Frozen spins}

If the spins are frozen, $\mathbf{S}_{\perp, \ell}$ do not depend on time, and we are left with a pure static $(\omega=0)$ contribution:

$$
\frac{\partial^{2} \sigma}{\partial \Omega \partial E_{f}}=\left(\gamma_{N} r_{o}\right)^{2} \delta(\omega) \sum_{\tau_{m}} \delta\left(\mathbf{Q}-\tau_{m}\right)\left|\mathbf{F}_{m}(\mathbf{Q})\right|^{2}
$$

$\tau_{m}$ is a reciprocal magnetic lattice vector and $\mathbf{F}_{m}$ is the magnetic structure factor:

$$
\mathbf{F}_{m}(\mathbf{Q})=\sum_{\ell} \mathbf{S}_{\perp, \ell} e^{i \mathbf{Q} \mathbf{r}_{\ell}} e^{-W_{\ell}} f_{\ell}(\mathbf{Q})
$$

\subsection{Spin waves}

In the spin wave approximation, the elementary excitations, called spin waves, correspond to precession motions of the spins around the magnetization direction [12-17]. These spin waves are free particles carrying a spin 1 . Tough calculations show that:

$$
\frac{\partial^{2} \sigma}{\partial \Omega \partial E_{f}}=\sum_{s} A_{s}\left[\left(1+n\left(\omega_{\mathbf{Q}, s}\right)\right) \delta\left(\omega-\omega_{\mathbf{Q}, s}\right)+n\left(\omega_{\mathbf{Q}, s}\right) \delta\left(\omega+\omega_{\mathbf{Q}, s}\right)\right]
$$

In close analogy with the case of phonons, the cross section takes the form of a sum of Dirac functions on the different spin waves branches. Again, this formula shows that an inelastic neutron scattering experiment allows a direct measurement of the dispersion 

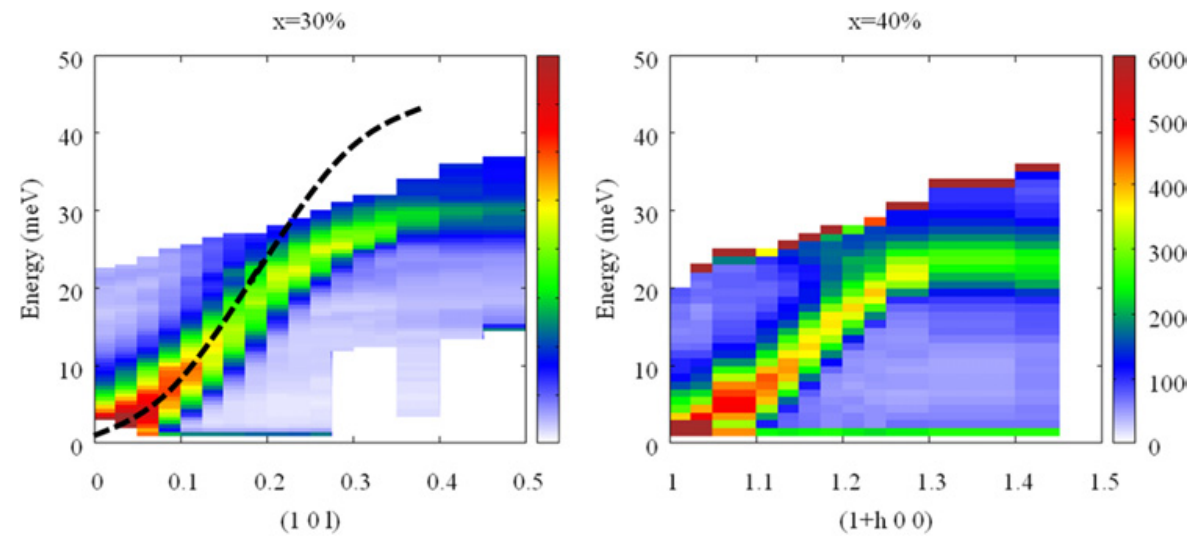

Figure 8. Spin wave dispersion in the cubic ferromagnet $\mathrm{La}_{1-x} \mathrm{Sr}_{x} \mathrm{MnO}_{3}$ compounds. A simple cosine dispersion is expected. Note the flattening close to the zone boundary which has not been explained yet despite a huge theoretical work.

relations. The intensity of the modes is given by a "dynamical" structure factor $A_{s}$, whose general expression, however, is rather complex; its derivation goes far beyond the scope of this lecture. Figure 8 shows the mapping measured for instance in the $\mathrm{La}_{1-x} \mathrm{Sr}_{x} \mathrm{MnO}_{3}$ compounds, very well know for their magneto-resistance properties. These systems are simple cubic ferromagnets (lattice parameter $a$ ), and one expects a dispersion relation reading as:

$$
\omega_{\mathbf{k}}=2 J S\left(3-\cos k_{x} a-\cos k_{y} b-\cos k_{z} a\right)
$$

As can be observed from Fig. 8, this simple form fits very well the experiment at small $\mathbf{q}$, but is clearly not adapted close to the zone boundary: the dispersion becomes rather flat, an effect which remains mysterious so far, not reproduced by theoretical calculations. This illustrates the relevance and simplicity of inelastic neutron scattering, which definitely provides valuable information about the spectrum of elementary excitations even in very complex condensed matter.

\subsection{Crystal field levels}

The response due to the inelastic scattering by crystal field excitations is also very useful in practice. The latter correspond to the different transition between states describing the electronic configurations of the (usually $4 \mathrm{f}$ or $5 \mathrm{f}$ ) electrons. Denoting $E_{n}$ and $\phi_{n}$ the energies and wave functions, we get:

$$
\begin{aligned}
\int_{-\infty}^{+\infty} d t\left\langle\mathbf{S}_{\perp, \ell} \mathbf{S}_{\perp, \ell}(t)\right\rangle e^{-i \omega t} & =\int_{-\infty}^{+\infty} d t \sum_{n, m} \frac{e^{-E_{n} / T}}{Z}\left\langle\phi_{n}\left|\mathbf{S}_{\perp, \ell}\right| \phi_{m}\right\rangle\left\langle\phi_{m}\left|\mathbf{S}_{\perp, \ell}\right| \phi_{n}\right\rangle e^{i\left(E_{m}-E_{n}-\omega\right) t} \\
& =\sum_{n, m} \frac{e^{-E_{n} / T}}{Z}\left|\left\langle\phi_{n}\left|\mathbf{S}_{\perp, \ell}\right| \phi_{m}\right\rangle\right|^{2} \delta\left(E_{m}-E_{n}-\omega\right) \\
Z & =\sum_{n} e^{-E_{n} / T}
\end{aligned}
$$


from which one deduces:

$$
\frac{\partial^{2} \sigma}{\partial \Omega \partial E_{f}}=\frac{k_{f}}{k_{i}}\left(\gamma_{N} r_{o}\right)^{2} \sum_{\ell}\left|f_{\ell}(Q)\right|^{2} e^{-2 W_{\ell}} \sum_{n, m} \frac{e^{-E_{n} / T}}{Z}\left|\left\langle\phi_{n}\left|\mathbf{S}_{\perp, \ell}\right| \phi_{m}\right\rangle\right|^{2} \delta\left(E_{m}-E_{n}-\omega\right)
$$

As a result, the scattering due to crystal field shows Dirac functions at all possible transitions between two eigenstates $\omega=E_{m}-E_{n}$. Note that there is no particular $Q$ dependence: indeed, no correlations are expected since the moments do not interact with each other, except the Debye Waller and the form factor. At low temperature, most of the possible transitions disappear since $\frac{e^{-E_{n} / T}}{Z} \approx \delta_{n, 1}$, so that we are left with the transition from the ground state only. The cross section becomes:

$$
\frac{\partial^{2} \sigma}{\partial \Omega \partial E_{f}}=\frac{k_{f}}{k_{i}}\left(\gamma_{N} r_{o}\right)^{2} \sum_{\ell}\left|f_{\ell}(Q)\right|^{2} e^{-2 W_{\ell}} \sum_{m}\left|\left\langle\phi_{1}\left|\mathbf{S}_{\perp, \ell}\right| \phi_{m}\right\rangle\right|^{2} \delta\left(E_{m}-E_{1}-\omega\right)
$$

\subsection{Metal}

In a metal, or by the way, in any material where the elementary excitations are fermions carrying a spin half (like spinons in 1D chains, for instance), the spin excitations is composed of a continuum that is nothing but a kind of two particles density of states. Indeed, since the particles carry a spin half, two "flips", hence a pair of particles, is necessary to match the $\Delta S=1$ transition of the neutron spin. For a given $(\mathbf{k}, \omega)$, different pairs of fermions contribute provided their energies $E_{\mathbf{q}}$ and $E_{\mathbf{q}^{\prime}}$ fulfill the condition $\omega=E_{\mathbf{q}^{\prime}}+E_{\mathbf{q}}$ and provided the momentum is conserved $\mathbf{k}=\mathbf{q}+\mathbf{q}^{\prime}$. The corresponding spectral weight depends however on details of the Hamiltonian [18].

\section{Fluctuation dissipation}

We would like now to close this lecture by pointing out the link between two equivalent languages: correlations and response function or generalized susceptibility [19]. Actually, the response of a system under a time dependent perturbation $B(t)$ results in the emergence of an observable $A$. Applying a magnetic field will for instance result in a magnetization, or applying a force will result in atomic displacements. In the framework of linear response theory, $A$ and $B$ are "proportional", related by a generalized susceptibility $G$ :

$$
A(t)=\int d t^{\prime} G\left(t-t^{\prime}\right) B\left(t^{\prime}\right)
$$

or in frequency representation:

$$
A(\omega)=G(\omega) B(\omega)
$$

where the Fourier transform $G(\omega)$ is defined as:

$$
G(\omega)=\lim _{\epsilon \rightarrow 0^{+}} \int d t e^{i \omega t-\epsilon t} G(t)
$$

The theory shows that [19]:

$$
G(t)=i \theta(t)\langle[A, B(t)]\rangle_{o}
$$

where the expectation value is to determined with the unperturbed Hamiltonian of the system $(B=0)$. If one denotes $E_{n}$ and $\phi_{n}$ the eigen-energies and wave functions of the system, one 
obtains:

$$
G(t)=i \theta(t) \sum_{n} \frac{e^{-E_{n} / T}}{Z}\left\langle\phi_{n}|[A, B(t)]| \phi_{n}\right\rangle_{o}
$$

Introducing $\left\langle\phi_{n}|A| \phi_{m}\right\rangle=A_{n m}$, and calculating the commutator, this becomes:

$$
G(t)=i \theta(t) \sum_{n, m} \frac{e^{-E_{n} / T}}{Z}\left(A_{n m} B_{m n} e^{i\left(E_{m}-E_{n}\right) t}-B_{n m} A_{m n} e^{-i\left(E_{m}-E_{n}\right) t}\right)
$$

or

$$
\begin{aligned}
G(t) & =i \theta(t) \sum_{n, m} \frac{e^{-E_{n} / T}}{Z} A_{n m} B_{m n} e^{i\left(E_{m}-E_{n}\right) t}-\frac{e^{-E_{m} / T}}{Z} B_{m n} A_{n m} e^{-i\left(E_{n}-E_{m}\right) t} \\
& =i \theta(t) \sum_{n, m} \frac{e^{-E_{n} / T}-e^{-E_{m} / T}}{Z} A_{n m} B_{m n} e^{i\left(E_{m}-E_{n}\right) t} \\
& =i \theta(t) \sum_{n, m} \frac{e^{-E_{n} / T}}{Z}\left(1-e^{-\left(E_{m}-E_{n}\right) / T}\right) A_{n m} B_{m n} e^{i\left(E_{m}-E_{n}\right) t}
\end{aligned}
$$

Using:

$$
\begin{aligned}
\lim _{\epsilon \rightarrow 0^{+}} \int d t e^{i \omega t-\epsilon t} i \theta(t) e^{i x t} & =\lim _{\epsilon \rightarrow 0^{+}} \frac{i[0-1]}{i(\omega+x)-\epsilon} \\
& =\lim _{\epsilon \rightarrow 0^{+}} \frac{-1}{(\omega+x)+i \epsilon} \\
& =-\operatorname{Pf} \frac{1}{\omega+x}+i \pi \delta(\omega+x)
\end{aligned}
$$

We have for the imaginary part of $G(\omega)$ :

$$
\begin{aligned}
G^{\prime \prime}(\omega) & =\pi \sum_{n, m} \frac{e^{-E_{n} / T}}{Z}\left(1-e^{-\left(E_{m}-E_{n}\right) / T}\right) A_{n m} B_{m n} \delta\left(\omega+\left(E_{m}-E_{n}\right)\right) \\
& =\pi\left(1-e^{-\omega / T}\right) \sum_{n, m} \frac{e^{-E_{n} / T}}{Z} A_{n m} B_{m n} \delta\left(\omega+\left(E_{m}-E_{n}\right)\right)
\end{aligned}
$$

On the other hand, correlations between $A$ and $B$ are defined by:

$$
C(t)=\langle A B(t)\rangle_{o}
$$

which can be rewritten as:

$$
C(\omega)=\sum_{n, m} \frac{e^{-E_{n} / T}}{Z} A_{n m} B_{m n} \delta\left(\omega+\left(E_{m}-E_{n}\right)\right)
$$

As a result:

$$
\begin{aligned}
& G(\omega)=\pi\left(1-e^{-\omega / T}\right) C(\omega) \\
& C(\omega)=\frac{1}{\pi} \frac{1}{1-e^{-\omega / T}} G(\omega)
\end{aligned}
$$


These calculations demonstrate the relations between the generalized susceptibility (also called response function) and the correlations. $\left(1-e^{-\omega / T}\right)^{-1}$ is called the detailed balance factor. The magnetic inelastic neutron cross section is a measure of correlations between spins; it is also nothing but the imaginary part of the frequency dependent magnetic susceptibility.

\section{Conclusion}

In this lecture, the basics of inelastic neutron scattering were presented. We have especially pointed out that diffraction measures instantaneous correlations, while inelastic scattering determine the time dependence of those correlations. This allows a direct measurement of the dispersion of elementary excitations, like phonons or spin waves.

\section{References}

[1] W. Szuszkiewicz, unpublished

[2] G.L. Squires, Introduction to the Theory of Neutron Scattering (Dover Publications, 1996)

[3] P.G. de Gennes, Theory of Neutron Scattering by Magnetic Crystals, in Magnetism, Vol. III, Eds. G.T. Rado and H. Suhl (New York: Academic Press, 1963), p.115-147

[4] Neutron Scattering, ed. by K. Sköld, D.L. Price, Methods in Experimental Physics, Vol. 23C (Academic Press, New York, 1987)

[5] S.W. Lovesey, Theory of Neutron Scattering from Condensed Matter, Vol 2 (Oxford Science Publications, 1984)

[6] Y.A. Izyumov and N.A. Chernoplekov, Neutron Spectroscopy (Plenum, 1994)

[7] B.N. Brockhouse. Inelastic scattering of neutrons in solids and liquids, Vienna. 1961. p. 113; http://nobelprize.org/nobel_prizes/physics/laureates/1994/ brockhouse-lecture.html

[8] G. Shirane, S.M. Shapiro and J. Tranquada, Neutron Scattering with a Triple-Axis Spectrometer, Basic Techniques (Cambridge University Press, 2002)

[9] Diffusion inélastique de neutrons par temps de vol. J. Ollivier et J.-M. Zanotti. JDN 16, Collection SFN 10,379-423 (2010) https://doi .org/10.1051/sfn/2010006

[10] La diffusion inélastique des neutrons sur monocristal. Le spectromètre 3-axes. B. Hennion. JDN 16, Collection SFN 10, 357-378 (2010) https://doi.org/10.1051/sfn/2010005

[11] Z. Yamani, Z. Tun, and D.H. Ryan, Can. J. Phys. 88, 771-797 (2010)

[12] Magnetic Excitations, W.G. Stirling et K.A. McEwen, Methods of Experimental Physics, Vol. 23 Part C (Academic Press, 1987)

[13] D.C. Mattis, Theory of Magnetism I (Springer Verlag, 1988)

[14] R.M. White, Quantum Theory of Magnetism (Springer Verlag, 1987)

[15] A. Auerbach, Interacting electrons and Quantum Magnetism (Springer Verlag, 1994)

[16] D.C. Wallace, PR 128, 1614 (1962) Spin waves in complex lattice

[17] Numerical simulations and magnetism. S. Petit, JDN 18, Collection SFN 12, 105-121 (2011) https://doi.org/10.1051/sfn/201112006

[18] Magnétisme de bande et Supraconductivité: l'apport de la diffusion inélastique des neutrons. Yvan Sidis, JDN 20, Collection SFN 13, 04003 (2014) https://doi.org/10.1051/sfn/20141304003

[19] Physique statistique hors d'équilibre: Processus irréversibles linéaires. N. Pottier (EDP Sciences, 2007) 